Even with the brass wheel having some weight on the bearings, the system has a very definite zero. The motion of the mirror for small displacements is almost critically damped.

The instrument may be dismantled by inserting two small wire hooks through the hose connections at the top to support the floats while the oil flows out at the bottom.

A gauge constructed as above described has been in use at this laboratory and has given entire satisfaction. When not in use a small piece of rubber tubing is connected from one inlet to the other to keep the dust out. With an ordinary $U$ gauge an accumulation of dirt invariably appears at the point where the menisci are read; with this gauge, however, the graduations on both the wheel and the vernier are immersed in the oil and always appear bright and clean.

\title{
A SPECIFIC GRAVITY APPARATUS FOR GASES.
}

\section{By E. J. Brady.}

The simplest method of determining the specific gravity of gas would be by means of a $U$ tube with liquid in the lower end. similar to the method used for determining the density of liquids. This method has not been used for gases, because of the extremely small differential pressure that would have to be measured to obtain accuracy. The pressure gauge in the preceding note makes the use of this method possible.

The apparatus devised consisted of a tall tube, through which the gas was passed, having its lower end connected with the precision gauge by means of a four-way cock. At the upper end there is a pilot flame, if the gases being measured are inflammable. The gases being measured passed through this vertical tube, which is opened to the air through a suitable burner at the top. A quarter turn of the cock cuts off the gas and connects this column of gas with the precision pressure gauge, which will immediately indicate a slight suction produced on the gauge, provided that the gas is lighter than the air. This cock may be turned a number of times to obtain check readings on the same sample of gas. Using the telescope and scale as described, we may graduate the scale to read in specific gravity direct. The gravity as shown by a tube of this kind would, of course, include any foreign matter, such as particles of dust or vapors. But, inasmuch as these 
materials assist in producing the differential pressure in the Pitot tube or the Venturi meter, they should be taken into consideration in applying the formula for these instruments.

Gases heavier than air, such as oxygen, carbon dioxide, etc., may also be measured, the deflection produced being in the opposite direction.

For more accurate work, where the absolute density of the gas is wanted, we might provide a vertical tube for air also. This air could be dried to eliminate the effect of moisture and the whole system surrounded by the constant temperature bath.

Where the density of hot gases relative to air are required, the vertical air pipe could be carried within the vertical gas pipe, so that the air would quickly assume the same temperature as the gas being measured. In many places a vertical tube of much greater height would be permissible. In this case the telescope and scale could be dispensed with and the differential pressure read from the Vernier direct.

Remarkable Pitting of Electro-plating. O. P. WAtrs. (Proceedings of the American Electrochemical Society, April 3-5, I9I9.) -When making some lead platings, heavy pitting suddenly and irregularly appeared. After considerable study of the cause, it was finally found to be due to air dissolving in the electrolyte while it was resting over night and cooling, which was then expelled as minute air bubbles on the work when the bath was heated up by the passage of the current. The microscopic bubbles clinging to the work, which had been left immersed in the bath, started the pitting. (J.W. R.) The difficulty was overcome by heating the solution to a little above its working temperature by means of a lead steam coil before resuming plating, whenever the tanks had been out of use for any considerable time.

Similar trouble by gas-pitting may be expected whenever a plating solution that is used for heavy deposits, and operates at an elevated temperature, stands idle and cold for many hours; but if there is a steady evolution of hydrogen from the cathode during normal operation of the bath, as with the cyanide copper or the brass solution, no trouble need be anticipated, for the stream of gas, ascending along the surface of the metal, will prevent bubbles from clinging, long enough to form pits in the plating. Pitting may be expected only when the rate of evolution of gas from the work is extremely slow. 